\title{
Crowding and visual search in high functioning adults with autism spectrum disorder
}

This article was published in the following Dove Press journal:

Clinical Optometry

23 October 2010

Number of times this article has been viewed

\author{
Paul A Constable 1,2 \\ Joshua A Solomon' \\ Sebastian B Gaigg ${ }^{2}$ \\ Dermot M Bowler ${ }^{2}$ \\ 'Department of Optometry, Applied \\ Vision Research Centre, City \\ University London, United Kingdom; \\ ${ }^{2}$ Department of Psychology, Autism \\ Research Group, City University \\ London, United Kingdom
}

Purpose: Individuals with autism spectrum disorder have demonstrated faster reaction times when searching for objects in a visual scene. One possible explanation for this observation is that the influence of crowding may not be as strong within this group compared to typically developing individuals.

Subjects and methods: We recruited 16 participants with and without a diagnosis of autism spectrum disorder in the age range of 20-58 years. The main experiments focused on determining the critical spacing for the correct identification of an ellipse's orientation in the periphery when flanked by two circles with $81 \%$ correctly identified. The second experiment was an attempt to replicate previous studies that had demonstrated superior visual search in autism using reaction time, set-size slopes and intercepts as measures of search efficiency and pre-attentive processes.

Results: There were no significant group differences in the critical spacings for the crowded ellipses in the periphery $(P=0.358)$ or in the elliptical discrimination thresholds $(P=0.477)$. In addition there were no significant differences between groups in reaction times $(P=0.083)$, accuracy $(P=0.658)$ and set-size slopes $(P=0.976)$, however the intercept for the set-size slope function was significantly lower for the comparison group $(P=0.016)$.

Conclusions: The individuals we tested demonstrated neither immunity to crowding nor any advantage in the visual search task. Therefore, we failed to confirm that enhanced discrimination underlies superiority in visual search in adults with high functioning autism spectrum disorder. This finding may be associated with the older age group investigated compared to previous studies and suggests that the underlying mechanism of superior visual search may not be a persistent feature of autism spectrum disorder.

Keywords: critical spacing, reaction time, discrimination, visual perception

\section{Introduction}

The term, autism spectrum disorder (ASD) covers a wide range of abilities and disabilities that are present in the three core domains of communication, imagination and reciprocal social interaction. ${ }^{1}$ The visual experience of an individual with ASD also differs from that of typical individuals. ${ }^{2}$ Abnormalities in this domain may account for some of the clinically defining features of this disorder. Children with ASD will often focus on small details, and report seeing features (eg, shapes, numbers or patterns) of a scene that are either overlooked or not contextually relevant to the typical observer. One could generalize and describe the visual perception of an individual with ASD as having a cubist's perspective on the world in the way that parts of objects may assume greater importance than the whole. Despite agreement that there are clear differences between observers with autism and typical development, the relevant empirical findings
Correspondence: Paul Constable City University, Department of Optometry, Northampton Square, London ECIV OHB, United Kingdom Tel +44207040 4334

Fax +44207040 8494

Email paul.constable.I@city.ac.uk 
regarding visual task performance have not always concurred. Consequently a coherent understanding of visual experience in ASD is still lacking (see Dakin and Frith ${ }^{3}$ and Simmons et $\mathrm{al}^{4}$ for reviews).

The main findings that indicate individuals with ASD process visual information differently stem from visual discrimination and search tasks on which such individuals demonstrate superior performance. In the embedded figure test (EFT), where participants are required to identify a simple figure/shape within a more complex figure, ASD children $^{5}$ and adults ${ }^{6}$ are more adept. Functional magnetic resonance imaging (fMRI) studies have attempted to elucidate the structural areas that differ in ASD compared to typical children whilst performing the EFT. In one study, greater activity in extrastriate and right primary visual cortex in ASD children and adolescents was present compared to the more typical activation of left parietal and pre-motor areas in the comparison group. However, there was no performance advantage on the EFT in the ASD group, just a difference in cortical areas utilized. The authors interpreted their findings as evidence for an altered pattern of cortical involvement during such a task. ${ }^{7}$ An earlier fMRI study also found differences in cortical activity in children with ASD. However, rather than extra visual cortical activity the authors found lowered overall involvement in the ASD group. ${ }^{8}$ These findings, although discordant in the localization of cortical activity provide evidence for differences in cortical involvement during a discrimination task in children with ASD.

Superiority on the EFT forms the basis on which several cognitive theories have been developed to help explain not only visual perception but also the broader ASD phenotype. One explanation, known as the enhanced perceptual functioning (EPF), posits that the central abnormality lies in the visual cortex at the perceptual level. ${ }^{2}$ An alternative model that draws upon elements of the EPF to describe ASD is the 'weak central coherence' (WCC). ${ }^{9}$ This model has undergone a recent revision to account for the findings of enhanced perception. ${ }^{10} \mathrm{WCC}$, as outlined by Happé and Frith, draws upon the interpretation of enhanced perception at the local level into difficulties assembling or seeing the global context. ${ }^{10}$ The WCC model suggests that ASD traits are the result of a different cognitive style that biases attention to fine detail rather than the 'big picture'. This style is evident in the manner in which ASD boys would focus on fine detail when copying a picture. ${ }^{11}$ Thus WCC is a style of operating rather than a cognitive deficit per se. This style may explain the high level of performance seen in ASD savants. ${ }^{12}$ Poor motion coherence thresholds sometimes evident in ASD give some support to a WCC account of $\mathrm{ASD},{ }^{13}$ where the global pattern of motion conveyed in a fraction of dots moving together is reduced. The findings of a superior visual search also support WCC as the default position for visual processing tends towards the local rather than the global perspective as demonstrated in several studies. ${ }^{14-17}$

Whilst WCC accounts for part of the behavior seen in ASD, and has moved from a central executive model to one where enhanced or priority is given to lower order local elements rather than the global picture. The consequence being that in ASD, the bias of visual perception is on the side of the component parts rather than the whole. ${ }^{10}$ Plaisted et $\mathrm{al}^{28}$ draw upon the WCC theory to explain the superiority seen in visual search. They contend that individuals with ASD are more able at processing features of an object thus enabling faster reaction times in search tasks. In contrast to WCC, the EPF model localizes the primary source of atypical visual processing in ASD at the level of V1 rather than within the 'higher' neurocognitive system. ${ }^{2}$ This model is supported by evidence for exaggerated processing of low-level visual information that facilitates the higher discriminatory abilities observed in the visual $^{18}$ and also the auditory ${ }^{19}$ domain.

One model of visual perception is the Reverse Hierarchy Theory as proposed by Hochstein \& Ahissar. ${ }^{20}$ In this model, visual perception is a constant interaction between lower and higher cortical regions. At one level, orientation selective neurons detect the features of a surface and are associated with the global feed forward pathway. The detection of boundaries occurs where there is a difference in the orientation of feature elements defined by orientation selective neurons. The detection of the border or edges is mediated by lateral inhibition between these neurons that share a similar orientation $^{21}$ and results in a relatively greater response when there is a greater contrast between the orientations of lines than when the lines have a similar orientation. Information regarding an object's boundary is then fed forward to higher cortical areas providing the general impression of what the object is. Reciprocal feedback projections to V1 then enable information to be processed regarding the texture within the boundaries, and help fill in the details, and scrutinize the object's properties in finer detail. ${ }^{22,23}$ Vandenbroucke et al ${ }^{24}$ have used this model to probe the relative balance of feedback, feed forward and horizontal connections in ASD. The authors used psychophysics ${ }^{25}$ and EEG recordings ${ }^{24}$ to study their relative contributions in a figure-ground segregation task. The overall findings supported an imbalance in favor of feedback with atypical horizontal connectivity. Therefore, in ASD individuals the imbalance would suggest that their 
visual style is one where the details of an object are more important than the overall shape.

Additional support for higher perceptual abilities in primary visual cortex was identified using orientation discrimination thresholds. In Bertone et al's ${ }^{26}$ study of first and second order orientation gratings in which a first order grating was defined by luminance and second order grating was defined by texture, the second order stimulus is more complex, as evidenced by longer reaction times and slower evoked potentials. ${ }^{26}$ Bertone et al found the primary difference in ASD was related to the complexity of the stimulus. The orientation discrimination thresholds were superior in the first order stimulus, ${ }^{18}$ but poorer with the second order stimulus. Thus, the low-level processing of spatial frequency, orientation and contrast within primary visual cortex, was superior in ASD and may be a function of weaker local connectivity between cortical columns which yield enhanced perception of local elements.

Another viewpoint on ASD is based upon a continuum of which autism merely represents one extreme 'male brain' perspective. ${ }^{27}$ In this model, an individual is inclined towards systemizing and understanding the whole by breaking it down into component parts. The extreme male brain theory is an alternative explanation to the behavioral characteristics of ASD. Its underlying premise is that altered levels of androgens affect neural development and results in an extreme 'male brain' pattern of behavior. Individuals with ASD often display systemizing patterns of behavior and this leaning towards detecting patterns may confer superiority in detecting fundamental features and may contribute to search superiority in ASD.

Visual search superiority in ASD has been demonstrated for both feature and conjunctive search tasks in adults ${ }^{17}$ and children. ${ }^{16,28}$ One explanation for this search superiority is that children with ASD are able to discriminate between items displayed with greater ease. When the target-distracter similarity increases, the reaction times of ASD children were faster than typically developing children. ${ }^{15}$ The question of whether memory or altered search strategies could contribute to the superior search times has also been investigated. Kemner et $\mathrm{al}^{29}$ measured eye-movements and reaction times in a group of adults with pervasive developmental disorder who failed to meet full diagnostic criteria for ASD but still showed impairments in social and language development. Inappropriate comma use; unnecessary definition of hard and easy tasks (these terms are never used again). In fact, the whole sentence seems superfluous. The important finding was that the PDD group made fewer saccades and had shorter fixation times whilst performing the task; implying that in these individuals they were able to detect the search item 'at a single glance', suggesting greater powers of discrimination as suggested by O'Riordan and Plaisted..$^{15}$ Another attempt to explore the basis of superior visual search also utilized eye-movement tracking as well as presenting the search displays as either 'static' or 'dynamic'. In this study by Joseph et $\mathrm{al}^{14}$ the static search had a letter T fixed amongst letter Ls as distracters, whilst in the dynamic display the arrangement of the target and distracters changed every 500 msecs to prevent the memory of prior search influencing the reaction times. The authors found the ASD group was faster in both static and dynamic search conditions than the comparison children. In addition, the authors found no differences between the groups on eye-movements performed during each search type, which excluded a different search strategy or memory involvement as factors that could account for the results. One additional aspect reported by Joseph et a ${ }^{14}$ was the set-size slopes and intercepts for the groups. As the number of distracters (set-size) in a search task increases then so does the reaction time. The set-size slope is a measure of search efficiency and the intercept is a measure of the speed of processing the features. Thus, a reduced intercept would indicate faster early perceptual processing in the ASD group, which the authors found. However, the set-size slopes were not different between groups, in the static and dynamic search conditions, which supported the eye-movement data indicating no difference in search strategy in ASD compared with the comparison group. The main conclusion was that the significant differential between ASD and comparison children was the early processing of visual information as implied by the lower intercepts of the set-size slope functions, suggesting superior pre-attentive or early processing was the basis for superior search ability.

Given several reports of a general bias to local compared to the global picture in discrimination tasks, then this finding should be consistent across all discrimination tasks if it is a defining feature of ASD. In a recent review Kaiser and Shiffrar ${ }^{30}$ addressed the issue of local advantage in the static discrimination tasks such as visual search and EFT, compared to the studies investigating local and global motion. If there is a bias for local over global processing then this bias should be present in the motion domain as well. For example, coherent motion thresholds are higher in ASD supporting the inability to connect the global pattern of motion ${ }^{31,13}$ thus supporting an impairment in global processing. However, these findings have not been consistently replicated using similar random dot arrays. ${ }^{32,33}$ Motion onset visual-evoked potentials (VEPs) do show variation with age ${ }^{34}$ and the differences found in motion 
detection may reflect differences in the development and maturation of magnocellular pathways in this group. To date no studies have utilized EEGs to ascertain what cortical regions are active during motion detection in ASD across a wide age range to ascertain how these processes may develop over time.

One suggestion to address the issues of perceptual discrimination is to assess how individuals with ASD perform in crowding. ${ }^{3}$ Crowding can be described as the inability to identify an object when other objects are nearby. ${ }^{35,36}$ One popular index of crowding is its critical spacing, which is the distance at which a distracter begins to influence target identification. According to Bouma's "law", identification of a target at retinal eccentricity (E) should remain unimpaired, as long as no other objects appear within E/2 of it. ${ }^{37}$ Results from two recent studies suggest that the efficiency of visual search is limited by crowding. ${ }^{38,39}$ A reduced susceptibility to visual crowding may be one reason why individuals with ASD outperform typical observers in visual search tasks. Despite crowding being an extensively studied area of visual perception, there has only been one study that has investigated this property in ASD children. ${ }^{40}$ In that study Baldassi et $\mathrm{al}^{40}$ used detection of the orientation of a tilted Gabor patch placed $6^{\circ}$ in the periphery and surrounded by eight vertically orientated Gabors. They found that, once correction for the discrimination threshold of the uncrowded Gabor was used, then the children with ASD were less susceptible to crowding.

In individuals with amblyopia, Bouma's "law" ${ }^{36}$ does not hold and the critical spacing is larger than that for normal observers. Furthermore, amblyopes have difficulty identifying targets at the fovea (ie, $E=0$ ) when other objects are nearby. ${ }^{35}$ Amblyopia is also a developmental disorder, just as ASD is, but in contrast to individuals with ASD amblyopes have difficulty with visual search. ${ }^{41}$ Imaging studies in amblyopia also indicate abnormalities in primary visual cortex that underlies this condition. ${ }^{42}$ Therefore, if in autism there is superiority in visual search, associated with higher discrimination of target features in primary visual cortex, then individuals with ASD may have smaller critical spacings. Our first goal was to determine the critical spacing for our ASD population compared to the comparison group to ascertain whether differences in the critical spacing were present.

The second goal was to replicate the findings of several groups who have shown faster reaction times in feature search tasks. The work of O'Riordan ${ }^{17}$ was used as a model, as she had demonstrated a clear superiority in the feature search condition in 10 closely matched individuals with an age range of 17-27 years either with a diagnosis of autism or without.
In O'Riordan's ${ }^{17}$ study she found superiority in two search tasks, one was conjunctive and the second was a feature search task. A conjunctive search task is more difficult to perform because the target and distracters share some attributes. The observer must decide on an item-by-item serial search whether the target is present or absent. O'Riordan did find superiority in the conjunctive search task in her group when letters were used, however, when letters were used as feature targets then this advantage was not seen and this was attributed to possible ceiling effects based upon the stimuli used. Therefore, O'Riordan used a different feature search stimulus to assess search superiority between the groups, namely an ellipse amongst circular distracters. The feature search task is the simplest task because the observer is required to decide whether a target is either present or absent based upon one feature that distinguishes the target from the distracters. O'Riordan found significantly faster reaction times in her group with autism compared to the comparison individuals. This finding indeed supported the previous work of O'Riordan and Plaisted ${ }^{15}$ who demonstrated the same search superiority in children. Therefore, we chose that same feature search task in order to mimic as closely as possible the stimuli used by O'Riordan. In addition, feature searches were also used in studies that implicated crowding in search. ${ }^{38,39}$

This study's aim was to investigate whether ASD adults were resistant to crowding in the periphery, and if crowding might therefore explain the advantages seen in visual search tasks in this group. This study is also the first to investigate these properties in an older population of ASD individuals and begins to address the issue of how stable visual perception may be in this group. There have been no longitudinal studies or test-retest studies to determine how reliable findings of search superiority are in ASD. There is paucity in the literature concerning the abilities in visual processing in adults with ASD over the age of 25 years. Whilst it is natural to ask, what differences occur during development in ASD it is also important to ask what changes there may or may not be in the cortical processes in these individuals over time?

\section{Material and methods \\ Apparatus}

For all experiments, an iMAC 7.1 computer running MATLAB $^{\mathrm{TM}}$ (MathWorks Ltd) was used for stimulus generation, experiment control and recording subjects' responses. The LCD monitor $(1680 \times 1050$ pixels at $75 \mathrm{~Hz})$ was $80 \mathrm{~cm}$ from the participants with mean luminance of $65 \mathrm{~cd} / \mathrm{m}^{2}$. The programs controlling the experiment incorporated elements of the PsychToolbox. ${ }^{43,44}$ 


\section{Stimuli}

A Gaussian blur kernel ( $\sigma=2$ pixels) was used for antialiasing the 1-pixel-wide ellipses and circles. A 2-pixel $\times$ 2-pixel fixation spot disappeared only during presentation of the search arrays in experiment 2. Ellipses and circles were white and the fixation spot was black. The circles used had a diameter of $1 \mathrm{~cm}$ (visual angle $0.72^{\circ}$ ) and the ellipse when present had a long axis of $1.2 \mathrm{~cm}$ and a short axis of $0.8 \mathrm{~cm}$ with a long axis visual angle of $0.86^{\circ}$ and short axis visual angle of $0.57^{\circ}$. The source codes for the stimuli are available upon request.

\section{Participants}

A total of 16 participants performed the visual search task and of these 14 performed the crowding task successfully. Two individuals in each group had unreliable critical spacings and were excluded from the analysis. The participants were all naïve to the task, but form a cohort of individuals that are regularly recruited by the department. All participants were matched for chronological age, verbal, performance and full intelligence quotient (IQ) as measured by the Wechsler Adult Intelligence Scale (WAIS-III ${ }^{\mathrm{UK}}$ ). ${ }^{45}$ Participants with ASD were diagnosed according to conventional criteria and a review of available medical records and assessment with the Autism Diagnostic Observational Schedule (ADOS) ${ }^{46}$ confirmed that all met Diagnostic and Statistical Manual of Mental Disorders, Fourth edition, Text Revision (DSM-IV$\mathrm{TR})^{47}$ criteria for ASD. Clinical diagnoses were made by local health authorities and/or experienced clinicians. Participants were also screened using the Autism Spectrum Quotient (AQ) with a cut off $<25$ required for the comparison group. ${ }^{48}$ See Tables 1 and 2 for participant details in each experiment. An unpaired $t$-test was used for statistical comparison between groups on age, full IQ, performance IQ, verbal IQ and AQ with $(P<0.05$ as significant). Individuals were paid standard University fees and gave their written and informed consent

Table I Participant details for Experiment I: Crowding (mean \pm SEM)

\begin{tabular}{llllll}
\hline & AGE years & FIQ & VIQ & PIQ & AQ \\
\hline ASD N $=14$ & $38.8 \pm 3.5$ & $108 \pm 4$ & $108 \pm 3$ & $107 \pm 4$ & $33 \pm 2$ \\
$\begin{array}{l}\text { (I2M : 2F) } \\
\text { Comparison }\end{array}$ & $43.5 \pm 3.2$ & $1 \mathrm{II} \pm 4$ & $107 \pm 4$ & $\mathrm{II} \pm 4$ & $\mathrm{II} \pm \mathrm{I}$ \\
$\mathrm{N}=\mathrm{I} 4$ & & & & & \\
$\begin{array}{l}\text { (IOM : 4F) } \\
P \text {-value }\end{array}$ & 0.334 & 0.625 & 0.898 & $0.65 \mathrm{I}$ & $<0.00 \mathrm{I}$ \\
\hline
\end{tabular}

Abbreviations: $\mathrm{FlQ}$, full intelligence quotient; $\mathrm{VIQ}$, verbal intelligence quotient; $\mathrm{PIQ}$, performance intelligence quotient; $\mathrm{AQ}$, autism quotient, SEM, standard error of the mean.
Table 2 Participant details for Experiment 2: Visual search (mean \pm SEM)

\begin{tabular}{llllll}
\hline & AGE years & FIQ & VIQ & PIQ & AQ \\
\hline $\begin{array}{l}\text { ASD N }=16 \\
(I 4 M: 2 F)\end{array}$ & $37.6 \pm 3.1$ & $110 \pm 4$ & $109 \pm 3$ & $109 \pm 4$ & $33 \pm 2$ \\
$\begin{array}{l}\text { Comparison } \\
\mathrm{N}=16\end{array}$ & $44.6 \pm 2.9$ & $111 \pm 3$ & $108 \pm 3$ & $111 \pm 4$ & $11 \pm 1$ \\
$\begin{array}{l}\text { II }: 4 \mathrm{~F}) \\
P \text {-value }\end{array}$ & 0.115 & 0.765 & 0.797 & 0.788 & $<0.00 \mathrm{I}$ \\
\hline
\end{tabular}

Abbreviations: $\mathrm{FIQ}$, full intelligence quotient; VIQ, verbal intelligence quotient; $\mathrm{PIQ}$, performance intelligence quotient; $\mathrm{AQ}$, autism quotient, SEM, standard error of the mean.

before taking part. The University Ethics committee granted approval for the study.

All observers wore their habitual correction if required and their corrected monocular acuities were N5 or greater for inclusion in the study. All experiments were performed using binocular vision. Exclusion criteria were if they had any history of ocular surgery, heterotropia or were currently using any systemic or ocular medication.

\section{Experiment I: crowding}

We adapted Toet and Levi's ${ }^{49}$ extremely rapid procedures for estimating critical spacing for use with ellipses like those in O'Riordan's experiment $2 .{ }^{17}$ In Phase I, an adaptive staircase (QUEST) $)^{50}$ was used to estimate the "threshold" elliptical eccentricity denoted $e$, at which $81 \%$ accuracy could be attained for identifying the orientation of an ellipse on the left of fixation. The orientation of each ellipse was either vertical or horizontal. Another randomly interleaved staircase estimated the threshold for an ellipse on the right of fixation. The elliptical eccentricity $e$ is defined so that: $e=\sqrt{ }\left[1-(\mathrm{b} / \mathrm{a})^{2}\right]$ with $\mathrm{b}$ and a representing the length of the short and long axes respectively. The value $e$ is equal to 0 for a circle and approaches 1 as the elliptical $e$ increases, thus a low value of $e$ represents minimal deviation from a circle.

In Phase II, the left and right ellipse's $e$ was $\sqrt{2}$ times the corresponding elliptical discrimination thresholds determined in Phase I. Each of these "target" ellipses was flanked by two equidistant circles; one on the left and another on the right (see Figure 1). QUEST was used to estimate the "critical" spacing between each side's target and its flanks, at which $81 \%$ accuracy could once again be attained for orientation identification. Ellipses were centered in both phases on the horizontal meridian, 240 pixels (roughly $5^{\circ}$ ) away from fixation. The critical spacing is expressed as a fraction of retinal eccentricity, denoted by E. Stimulus duration was 0.12 seconds. To minimize after images, a full-field random-texture postmask was displayed for 0.4 seconds following each trial. 


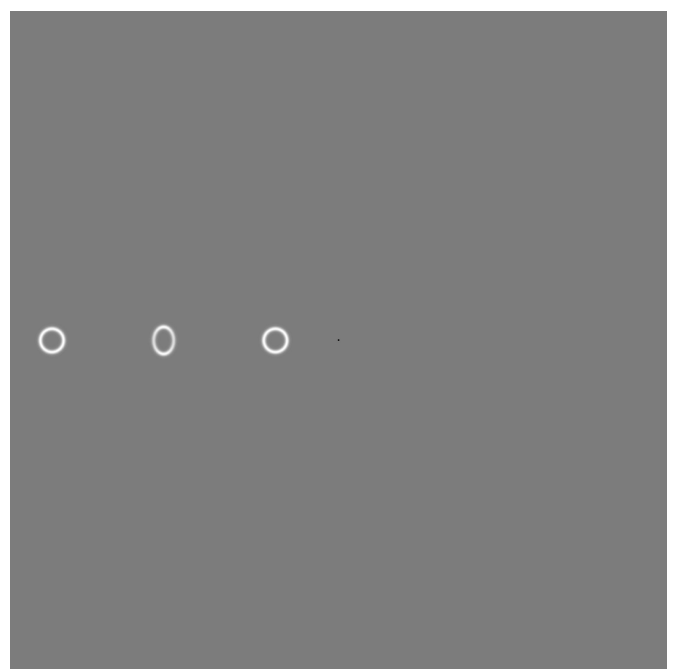

Figure I Crowded ellipse in the periphery. An example stimulus from Phase II of experiment one. In this example, the target ellipse has a vertical orientation. Its center has a retinal eccentricity $(E)$ of roughly $5^{\circ}$. The elliptical eccentricity (e) in this case is 0.39 . The distance between the central ellipse and circular flankers is the critical spacing and is expressed as a fraction of $E$.

Abbreviations: e, elliptical eccentricity; E, retinal eccentricity.

\section{Experiment 2: visual search}

The protocol for the feature search task adhered closely to that of O'Riordan (2004). For this task, observers were asked to indicate whether an ellipse was present or absent amongst a series of circular distracters using the keyboard input. The display either consisted of 4, 16 or 24 elements with the target ellipse being present or absent. The elements were arranged randomly on a $12 \times 12$ grid, so that an equal number appeared to the left and right of fixation (see Figure 2). Participants were asked to complete the task as quickly and as accurately as possible. The presentation was random so that the observer could not predict either the number of elements or whether an ellipse would be present or not. For each participant the

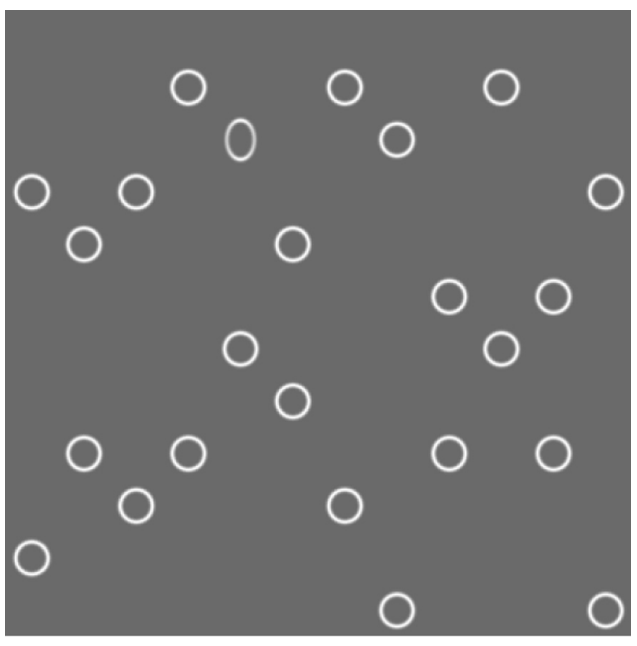

Figure 2 Target present set-size 24. A target-present search array with 23 distracters and I ellipse present from experiment two (Set-size $=24)$. first 12 trials were discarded as practice. A beep sounded if an error was made during any trial and this and the subsequent trial were not included in the analysis. Searches were also excluded from analysis if they took longer than 10 seconds. The reaction time and accuracy data were averaged for each participant, using the first 15 correct trials for each combination of set-size and target.

\section{Results}

\section{Experiment I: crowding}

The first phase of experiment one was to estimate the threshold elliptical eccentricity $e$ for an ellipse in the periphery. The mean $e$ thresholds for the left and right visual field were analyzed using the two-tailed unpaired $t$-test. All values are expressed as mean \pm standard error of the mean (SEM) with a total of 14 participants in each group successfully completing this task. The threshold elliptical eccentricity $(e)$ for the ASD group was $0.277 \pm 0.019$ and for the comparison group it was $0.257 \pm 0.020(\mathrm{t}(26)<1.0$; $P=0.477$ ) indicating that both groups were matched in their ability to discriminate between an ellipse and a circle in the periphery when it was not flanked by circular distracters. In Phase II, our main goal was to establish the critical spacings, of two equidistant flanking circles at which the orientation of the central ellipse could be determined with $81 \%$ certainty. The critical spacing is expressed as a proportion of retinal eccentricity E. For the ASD group the critical spacing was $0.640 \mathrm{E} \pm 0.023$. For the comparison group it was $0.596 \mathrm{E} \pm 0.041$ which was again, not significantly different $(\mathrm{t}(26)<1.0 ; P=0.358)$.

These results indicate that there is no difference between the observers in either their elliptical discrimination thresholds or importantly the critical spacing with both groups agreeing empirically with Bouma's law of $\sim 0.5 \mathrm{E}$.

\section{Experiment 2: visual search Reaction time analysis}

The mean reaction time (RT) data were analyzed using a Three-Way mixed analysis of variance (ANOVA) using the generalized linear model (GLM) procedure in Minitab ${ }^{\circledR}$ version 13.32 (Minitab, Pasadena, USA), with one between-subject factor of group (ASD or comparison) and two within-subject factors of target (present or absent) and set-size (4, 16 and 24) with a total of 16 participants in each group for this task. The ANOVA analysis revealed that the main effect of group was not significant $(\mathrm{F}(1,30)=3.04 ; P=0.083)$. Therefore, the group of ASD participants in this study did not display faster overall RTs in the search tasks compared to the comparison group. In our population the overall (mean \pm SEM) RTs for 
the comparison group $(1519 \pm 79 \mathrm{msec})$ were faster than the ASD group (1653 $\pm 71 \mathrm{msec})$.

The group by target interaction was not significant $(\mathrm{F}(1,30)<1.0 ; P=0.53)$, meaning there was no evidence of average RTs for ASD participants and the comparison group differing at all when the target ellipse was either absent or present. The group by set-size interaction was not significant $(\mathrm{F}(2,60)<1.0 ; P=0.95)$, meaning there was no evidence of average RTs for the ASD participants and comparison group differing at all across the 3 set-sizes (see Figure 3).

Further aspects of our results applied regardless of group. These main effects and interactions all replicated standard visual search results ${ }^{51,52}$ and were consistent with the findings of O'Riordan (2004). There were significant main effects of target $(\mathrm{F}(1,30)=60.0)$ and set-size $(\mathrm{F}(2,60)=54.2)$. There was also a significant interaction between target and set-size $(\mathrm{F}(2,60)=7.86 ; P=0.001)$.

\section{Set-size slope size analysis}

The set-size slope is an estimate of the search efficiency represented by the slope of the RT versus set-size whilst the intercept is associated with early pre-attentive processing time..$^{53}$ Thus the set-size slope is an indication of search efficiency whilst the intercept is a guide to early perceptual processing of the targets. Set-size slopes and intercepts were analyzed for the 16 participants in each group that completed the visual search task. The mean \pm SEM slopes for the target present condition were $30.8 \pm 4.2 \mathrm{msec} /$ item for the ASD group and $28.7 \pm 2.9 \mathrm{msec} /$ item for the comparison group. The intercepts were $928 \pm 55 \mathrm{msec}$ for the ASD and $776 \pm 34 \mathrm{msec}$ for the comparison group. In the target absent condition the slopes were $65.8 \pm 7.8 \mathrm{msec} / \mathrm{item}$ for the ASD and $67.6 \pm 11.4 \mathrm{msec} /$ item for the comparison group and the intercepts were $962 \pm 70 \mathrm{msec}$ for the ASD and $851 \pm 45 \mathrm{msec}$ for the comparison group. See Figure 4.

A two-way ANOVA with one between subject factor of group (ASD or comparison) and one between factor of probe (present or absent) was performed. Analysis revealed that the slopes were significantly steeper in the probe absent than for probe present trials $\mathrm{F}(1,30)=25.02 ; P<0.001$ although there was no group differences $\mathrm{F}(1,30)<1.0 ; P=0.976$. However, the intercepts were significantly different between groups $\mathrm{F}(1,30)=6.02 ; P=0.017$ with the ASD group showing higher intercepts in contrast to Joseph et al's (2009) study in which the ASD group exhibited lower intercept values.

\section{Accuracy analysis}

The average error score over trials was analyzed in the same manner as the average RT data, using a three-way mixed ANOVA with one between-subject factor of group (ASD or comparison) and two within-subject factors of target (present or absent) and set-size (4, 16 and 24) with 16 participants in each group.

In agreement with O'Riordan's participants, there was no overall difference in the number of errors made by each group $(\mathrm{F}(1,30)<1 ; P=0.658)$. The mean \pm SEM percentage error for the control group was $4.88 \% \pm 0.95 \%$ against $5.33 \% \pm 0.80 \%$ for the ASD group. There was no group by target interaction effect with the average percent errors $(\mathrm{F}(1,30)<1.0 ; P=0.35)$, meaning there was no systematic difference in the number of errors by the groups whether the target was absent or present. There was no group by set-size effect $(\mathrm{F}(2,60)<1.0$; $P=0.61)$ suggesting that average error rates between the two groups of participants were similar regardless of the experimental condition (see Figure 5). Once again in accordance with standard findings ${ }^{51,52}$ this error analysis also revealed a significant main effect of target $(\mathrm{F}(1,30)=50.4)$.
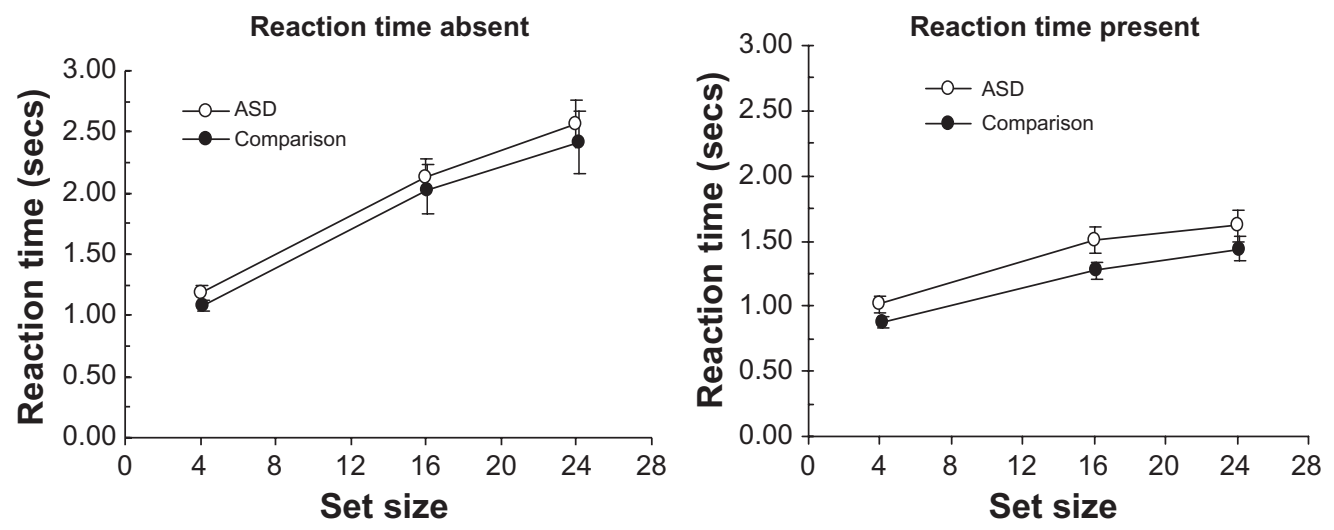

Figure 3 Reaction times for target absent and present. RT data for visual search of an ellipse amongst circles with set-sizes of 4 , 16 or 24 with the target ellipse either absent or present. Results are mean \pm SEM. The comparison group's data are offset slightly for clarity.

Abbreviations: RT, reaction time; SEM, standard error of the mean. 

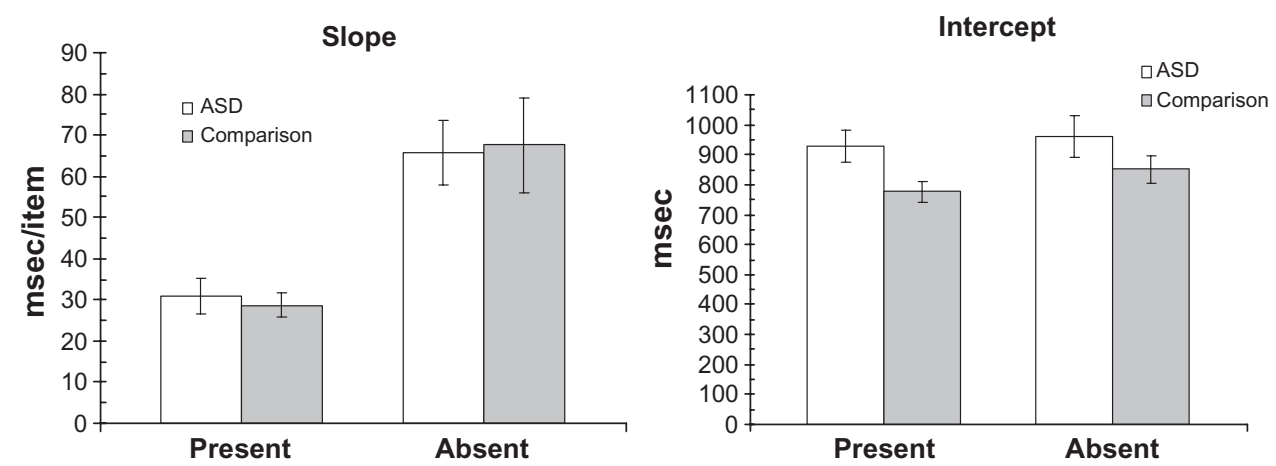

Figure 4 Set-size slopes and intercept plots. Comparison of set-size slopes and intercepts for the ASD and comparison group. The intercepts for the comparison group were significantly lower $(P=0.017)$. Error bars are mean \pm SEM.

Abbreviations: ASD, autism spectrum disorder; SEM, standard error of the mean.

\section{Age and task performance}

The large range of ages used in this study did not reveal any significant correlations with measures of task performance, indicating that age was not a predictor of performance in these individuals for both experiments. The Pearson correlation coefficient for age and elliptical discrimination threshold was 0.188 ; $P=0.551$ and for critical spacing it was $0.243 ; P=0.214$. For visual search tasks there was again, no correlation between age and the mean RTs across set-sizes for probe present $(0.183$; $P=0.315)$ or probe absent $(0.152 ; P=0.408)$. Similarly age was not correlated with the set-size slopes in the probe present $(0.232 ; P=0.202)$ or probe absent condition $(0.209, P=0.251)$ as well as the intercepts in the probe present $(0.036 ; P=0.844)$ or probe absent condition $(-0.127 ; P=0.488)$.

\section{Discussion}

The consensus of findings relating to visual search is that individuals with autism are faster and this is due to enhanced perception in primary visual cortex. This is a consistent finding across ages from childhood to early adulthood, and by extension, we presumed that these findings would be replicable in an older group of individuals with ASD. By addressing the issue of crowding, we sought to further increase our understanding of the visual abilities and disabilities seen in this group and relate these to visual search performance by replicating O'Riordan's (2004) feature-search experiment. The psychophysical results of our study found no differences between groups in their elliptical discrimination thresholds, nor the critical spacing related to crowding. Additionally we failed to demonstrate an advantage in a feature visual search task with respect to RT, accuracy and set-size slopes. However, in contrast to previous findings we found an increase in the intercept of the set-size slope function in the ASD group. The older ages of participants in this study group suggests that differences seen in younger individuals with ASD may not be as apparent in later life.
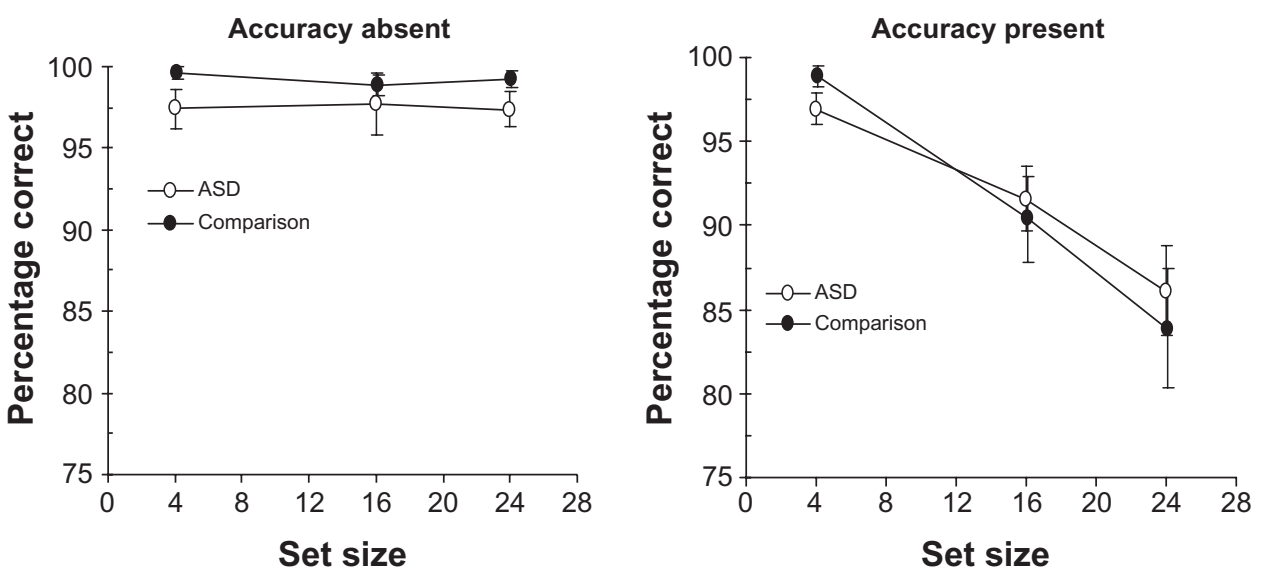

Figure 5 Accuracy for target absent and present. Accuracy data (mean \pm SEM) for target present or absent for the ASD and comparison group with set-sizes of 4 , 16 or 24. Note truncated Y-axis. The comparison group's data are offset slightly for clarity.

Abbreviations: SEM, standard error of the mean; ASD, autism spectrum disorder. 
The initial results indicated that between these high functioning adults and matched comparison group there were no significant differences in their elliptical discrimination thresholds or their susceptibility to crowding. This may be surprising in light of a recent report that ASD children may be immune to crowding. ${ }^{40}$ Unlike normal observers (eg, Parkes et al ${ }^{54}$ ) Baldassi et al's ${ }^{40}$ ASD observers did not find it more difficult to identify the tilt of a peripherally viewed Gabor when it was surrounded by untilted flanks. However, those children were significantly worse at identifying the tilt of unflanked Gabors (their average thresholds were 2.9 times higher), when compared to typically developing children. Thus, while those ASD children might be technically immune to crowding, their performances were not in any way superior to those of the comparison group given their significantly worse discrimination thresholds. In comparison, the adults used in this study showed no differences in their elliptical discrimination thresholds and this similarity between the groups may explain the different findings. It was only when the relative orientation discrimination thresholds were used that immunity to crowding was found in the Baldassi study. ${ }^{40}$

Despite considerable research into the properties of crowding, there is no absolute model that can account for these phenomena. ${ }^{35}$ Two current models propose different mechanisms with one proposing that crowding is a result of a reduction in the ability to combine local information across the receptive fields. ${ }^{54}$ The critical spacing represents the area over which local features are pooled. This pooling of features ultimately limits target detection based upon the stimulus's physical properties such as luminance, chromaticity and spatial frequency. In an alternative model, crowding may be a result from the finite limits of attention available to process information regarding the target and distracter. In the uncrowded condition, the target is readily identifiable, owing to the lower demands of attention required ${ }^{55}$ The notion that crowding is a result of perceptual integration rather than having a defined neural correlate, suggests that in these ASD observers there may be a shift in their capacity to broaden their focus of attention that is not as evident in the younger ASD populations tested to date.

In relation to the visual search task, in our high functioning adult group, we found no significant differences in RTs when the target was either present or absent in contrast to O'Riordan's findings. ${ }^{17}$ This was unexpected given that this finding has been replicated recently, although again in children aged 14 years and with a lower IQ (99) than the high functioning adults used in the present study. ${ }^{14}$ There are no studies reporting findings in adults over 30 with high functioning ASD and their performance on embedded figures or visual search nor are there any follow up longitudinal studies to see how these parameters may alter over time. With the increasing number and aging population of individuals with ASD, it will be of interest to see how their cortical functions develop over a lifetime. There were some differences in the average reaction times in our participants compared to O'Riordan's cohort. ${ }^{17}$ This is unlikely to be related to the size of the stimuli used as Joseph et $\mathrm{a}^{14}$ cohort also showed slower overall RTs using a similar sized stimulus to O'Riordan's study. ${ }^{17}$

Not all studies have replicated the features reported for visual search and the EFT in children with ASD. For instance, superiority in EFT is not apparent when children with high functioning autism/Asperger syndrome are tested. ${ }^{56}$ Baldassi et a ${ }^{40}$ also reported no differences in search ability when using discrimination thresholds rather than RTs as a measure of search efficiency. One group has challenged the EPF mode ${ }^{59}$ by investigating an older population. In their study, the authors used high functioning adults with ages ranging from 20-62 years to see if the higher discrimination ability was related to an inability to perceive differences between similar objects based upon a prototype of the object in question. The hypothesis being that if individuals with high functioning autism do have greater visual discrimination then this would be manifested by a reduction in their ability to categorize an object according to its prototype because they would perceive differences in every object and assign it to a novel category. The main findings were that these high functioning adults showed no reduction in the perceptual sensitivity based on their ability to categorize the items correctly. ${ }^{57}$ The authors concluded that enhanced discrimination is not a feature of ASD in older individuals. Certainly our data do not suggest any superiority in the early cortical processes. Indeed the intercepts of the set-size slope functions were significantly worse than the comparison group despite showing similar set-size slopes. This may mean that pre-attentive processes that are associated with the intercept are not heightened or superior in an older population and may reflect a change in the nature in which they attend to sensory stimuli over time.

The main difference between the participants in this study and earlier reports of superior visual search is the participants' ages. This may imply that despite neuroanatomical evidence for alterations in cortical structure ${ }^{58}$ and imaging studies that show over time the cortical volume, 
thickness and surface differ in $\mathrm{ASD}^{59}$ these early structural alterations do not necessarily impair function throughout life. The child with autism can and does develop social and communication skills, with alternative strategies, whilst these processes are delayed and/or rigid in their nature, they can be learnt through experience and the utilization or recruitment of alternative neural networks to compensate. The visual search and crowding data in this study suggest that despite evidence in the literature for superior search in childhood, that this aspect is not preserved throughout life. The study of Baldassi et $\mathrm{al}^{40}$ would also suggest that search superiority is not omnipresent in ASD. Indeed the findings here suggest that over time the local bias seen in childhood shifts towards the global perspective, and the cubists' abstraction begets a more composed and natural integration of the visual world.

\section{Conclusion}

With relation to models of visual processing in ASD, the data here provide evidence for normal psychophysical responses with relation to critical spacing and discrimination thresholds. This would suggest that the phenomenon of crowding does not contribute to the visual differences seen in this high functioning older ASD group. The normal elliptical discrimination threshold in these adults also suggests that their low-level visual processes are not affected on this measure. The most likely explanation is that any superiority seen in visual search is not 'hardwired' into the visual cortex in ASD. An individual with time, will experience and learn appropriate behavior and/ or responses to stimuli and it may be that with age, in ASD, comes the experience of where and how to focus one's attention and this ultimately diminishes the atypical visual patterns of perception noted in younger children and adolescents.

\section{Acknowledgments}

The City University, London pump priming fund, supported this work. PC is supported by a College of Optometrists, UK Research Fellowship. Parts of this study appeared in abstract form at IMFAR, Chicago 2009. The authors are grateful to the two anonymous reviewers of this work whose suggestions have significantly improved this manuscript. The authors would like to thank all participants for their time and co-operation during this study.

\section{Disclosure}

The authors report no conflicts of interest in this work.

\section{References}

1. Volkmar F, Chawarska K, Klin A. Autism in infancy and early childhood. Ann Rev Psychol. 2005;56(1):315-336.

2. Mottron L, Dawson M, Soulières I, Hubert B, Burack J. Enhanced perceptual functioning in autism: an update, and eight principles of autistic perception. J Autism Dev Disord. 2006;36(1):27-43.

3. Dakin S, Frith U. Vagaries of visual perception in autism. Neuron. 2005;48(3):497-507.

4. Simmons DR, Robertson AE, McKay LS, Toal E, McAleer P, Pollick FE. Vision in autism spectrum disorders. Vis Res. 2009;49(22): 2705-2739.

5. Shah A, Frith U. An islet of ability in autistic children: a research note. J Child Psychol Psychiat. 1983;24(4):613-620.

6. Jolliffe T, Baron-Cohen S. Are people with autism and Asperger syndrome faster than normal on the Embedded Figures Test? J Child Psychol Psychiatry. 1997;38(5):527-534.

7. Manjaly ZM, Bruning N, Neufang S, et al. Neurophysiological correlates of relatively enhanced local visual search in autistic adolescents. Neuroimage. 2007;35(1):283-291.

8. Lee PS, Foss-Feig J, Henderson JG, et al. Atypical neural substrates of Embedded Figures Task performance in children with Autism Spectrum Disorder. Neuroimage. 2007;38(1):184-193.

9. Happé F. Autism: cognitive deficit or cognitive style? Trends Cog Sci. 1999;3(6):216-222.

10. Happé F, Frith U. The weak coherence account: detail-focused cognitive style in autism spectrum disorders. J Autism Dev Disord. 2006;36(1): $5-25$.

11. Booth R, Charlton R, Hughes C, Happé F. Disentangling weak coherence and executive dysfunction: planning drawing in autism and attention-deficit/hyperactivity disorder. Philos Trans R Soc Lon B Biol Sci. 2003;358(1430):387-392.

12. Happé F, Frith U. The beautiful otherness of the autistic mind. Philos Trans R Soc Lon B Biol Sci. 2009;364(1522):1345-1350.

13. Pellicano E, Gibson L, Maybery M, Durkin K, Badcock DR. Abnormal global processing along the dorsal visual pathway in autism: a possible mechanism for weak visuospatial coherence? Neuropsychologia. 2005;43(7):1044-1053.

14. Joseph RM, Keehn B, Connolly C, Wolfe JM, Horowitz TS. Why is visual search superior in autism spectrum disorder? Dev Sci. 2009;12(6): 1083-1096.

15. O'Riordan M, Plaisted K. Enhanced discrimination in autism. Q J Exp Psychol A. 2001;54(4):961-979.

16. O'Riordan MA, Plaisted KC, Driver J, Baron-Cohen S. Superior visual search in autism. $J$ Exp Psycho Hum Percept Perform. 2001;27(3): 719-730.

17. O'Riordan MA. Superior visual search in adults with autism. Autism. 2004;8(3):229-248.

18. Bertone A, Mottron L, Jelenic P, Faubert J. Enhanced and diminished visuo-spatial information processing in autism depends on stimulus complexity. Brain. 2005;128(Pt 10):2430-2441.

19. Bonnel A, Mottron L, Peretz I, Trudel M, Gallun E, Bonnel A-M. Enhanced pitch sensitivity in individuals with autism: a signal detection analysis. J Cogn Neurosci. 2003;15(2):226-235.

20. Hochstein S, Ahissar M. View from the top: hierarchies and reverse hierarchies in the visual system. Neuron. 2002;36(5):791-804.

21. Knierim JJ, van Essen DC, Neuronal responses to static texture patterns in area V1 of the alert macaque monkey. J Neurophysiol. 1992; 67(4):961-980.

22. Lamme VAF. The neurophysiology of figure-ground segregation in primary visual cortex. J Neurosci. 1995;15(2):1605-1615.

23. Lamme VAF, Rodriguez-Rodriguez V, Spekreijse H, Separate processing dynamics for texture elements, boundaries and surfaces in primary visual cortex of the macaque monkey. Cereb Cortex. 1999;9(4): 406-413.

24. Vandenbroucke MWG, Scholte HS, Engeland Hv, Lamme VAF, Kemner C. A neural substrate for atypical low-level visual processing in autism spectrum disorder. Brain. 2008;131(Pt 4):1013-1024. 
25. Vandenbroucke MWG, Scholte HS, Engeland Hv, Lamme VAF, Kemner trum Disorder (ASD): investigating visual feedforward, horizontal and feedback processing. Vis Res. 2009;49(9):1006-1016.

26. Chakor H, Bertone A, McKerral M, Faubert J, Lachapelle P. Visual evoked potentials and reaction time measurements to motion-reversal luminance- and texture-defined stimuli. Doc Ophthalmol. 2005;110(2): $163-172$.

27. Baron-Cohen S. The extreme male brain theory of autism. Trends Cogn Sci. 2002;6(6):248-254.

28. Plaisted K, O'Riordan M, Baron-Cohen S. Enhanced visual search for a conjunctive target in autism: a research note. $J$ Child Psychol Psychiatry. 1998;39(5):777-783.

29. Kemner C, Ewijk Lv, Engeland Hv, Hooge I. Brief report: eye movements during visual search tasks indicate enhanced stimulus discriminability in subjects with PDD. J Autism Dev Disord. 2008;38(3): 553-557.

30. Kaiser MD, Shiffrar M. The visual perception of motion by observers with autism spectrum disorders: a review and synthesis. Psychon Bull Rev. 2009;16(5):761-777.

31. Milne E, Swettenham J, Hansen P, Campbell R, Jeffries H, Plaisted K. High motion coherence thresholds in children with autism. J Child Psychol Psychiatry. 2002;43(2):255-263.

32. de Jonge MV, Kemner C, de Haan EH, Coppens JE, van den Berg TJTP, van Engeland H. Visual information processing in high-functioning individuals with autism spectrum disorders and their parents. Neuropsychology. 2007;21(1):65-73.

33. Del Viva MM, Igliozzi R, Tancredi R, Brizzolara D. Spatial and motion integration in children with autism. Vision Res. 2006;46(8-9): 1242-1252.

34. Langrová, J, Kuba, M, Kremlácek J, Kubová Z, Vít F. Motion-onset VEPs reflect long maturation and early aging of visual motion-processing system. Vision Res. 2006;46(4):536-544.

35. Levi DM, Crowding - an essential bottleneck for object recognition: a mini-review. Vision Res. 2008;48(5):635-654.

36. Pelli DG, Tillman KA. The uncrowded window of object recognition. Nat Neurosci. 2008;11(10):1129-1135.

37. Bouma H. Interaction effects in parafoveal letter recognition. Nature. 1970;226(5241):177-178.

38. Gheri C, Morgan M, Solomon JA. The relationship between search efficiency and crowding. Perception. 2007;36(12):1779-1787.

39. Wertheim AH, Hooge ITC, Krikke K, Johnson A. How important is lateral masking in visual search? Exp Brain Res. 2006;170(3):387-402.

40. Baldassi S, Pei F, Megna N, et al. Search superiority in autism within, but not outside the crowding regime. Vision Res. 2009;49(16): 2151-2156.

41. Neri P, Levi DM. Spatial resolution for feature binding is impaired in peripheral and amblyopic vision. J Neurophysiol. 2006;96(1):142-153. C. A new approach to the study of detail perception in Autism Spec-

42. Bin L, Huiguang H, Xingfeng L, et al. Structural and functional deficits in human amblyopia. Neurosci Letts. 2008;437(1):5-9.

43. Brainard DH, The Psychophysics Toolbox. Spatial Vision. 1997;10: 433-436.

44. Pelli DG, The VideoToolbox software for visual psychophysics: transforming numbers into movies. Spatial Vision. 1997;10:437-442.

45. The Psychological Corporation. Wechsler Adult Intelligence Scale III UK edition. London, UK; 2000.

46. Lord C, Rutter M, Goode S, et al. Autism diagnostic observation schedule: a standardized observation of communicative and social behavior. J Autism Dev Disord. 1989;19(2):185-212.

47. American Psychiatric Association. Diagnostic and statistical manual of mental disorders, Fourth Edition, Text Revision (DSM-IV-TR); Washington DC, USA; 2000.

48. Baron-Cohen S, Wheelwright S, Skinner R, Martin J, Clubley E. The autism-spectrum quotient (AQ): Evidence from Asperger syndrome/ high-functioning autism, males and females, scientists and mathematicians. J Autism Dev Disord. 2001;31(1):5-17.

49. Toet A, Levi D. The two-dimensional shape of spatial interaction zones in the parafovea. Vision Res. 1992;32(7):1349-1357.

50. Watson AB, Pelli DG. QUEST: a Bayesian adaptive psychometric method. Percept Psychophys. 1983;33(2):113-120.

51. Duncan J, Humphreys GW. Visual search and stimulus similarity. Psychol Rev. 1989;96(3):433-458.

52. Treisman AM, Gelade G. A feature-integration theory of attention. Cogn Psychol. 1980;12(1):97-136.

53. Sternberg S. High-speed scanning in human memory. Science. 1966; 153(736):652-654.

54. Parkes L, Lund J, Angelucci A, Solomon JA, Morgan M. Compulsory averaging of crowded orientation signals in human vision. Nat Neurosci. 2001;4(7):739-744.

55. Intriligator $\mathrm{J}$, Cavanagh $\mathrm{P}$. The spatial resolution of visual attention. Cogn Psychol. 2001;43(3):171-216.

56. Kaland N, Mortensen EL, Smith L. Disembedding performance in children and adolescents with Asperger syndrome or high-functioning autism. Autism. 2007;11(1):81-92.

57. Bott L, Brock J, Brockdorff N, Boucher J, Lamberts K. Perceptual similarity in autism. $Q J$ Exp Psychol(Colchester). 2006;59(7): 1237-1254.

58. Casanova M, Trippe J. Radial cytoarchitecture and patterns of cortical connectivity in autism. Philos Trans R Soc Lond B: Biol Sci. 2009; 364(1522):1433-1436.

59. Raznahan A, Toro R, Daly E, et al. Cortical anatomy in autism spectrum disorder: an in vivo MRI study on the effect of age. Cereb Cortex. 2010; 20:1332-1340.

\section{Dovepress}

\section{Publish your work in this journal}

Clinical Optometry is an international, peer-reviewed, open access journal publishing original research, basic science, clinical and epidemiological studies, reviews and evaluations on clinical optometry. All aspects of patient care are addressed within the journal as well as the practice of optometry including economic and business analyses. Basic and clinical

research papers are published that cover all aspects of optics, refraction and its application to the theory and practice of optometry. The manuscript management system is completely online and includes a very quick and fair peer-review system, which is all easy to use. Visit http://www.dovepress. com/testimonials.php to read real quotes from published authors. 